\title{
REVIEW
}

\section{Risk Assessment of QT Prolongation with Citalopram and Escitalopram: An Evidence Based Review}

\author{
André S. Pollmann', Joel C. Bergman', Katie L. Lines ${ }^{1}$ \\ 'BSC Pharm canditate, College of Pharmacy, Faculty of Health Professions, Dalhousie University
}

\begin{abstract}
Recent health advisories have identified a risk of QT prolongation with the commonly used antidepressants citalopram (Celexa $\left.{ }^{\circledR}\right)$ and escitalopram (Cipralex $\left.{ }^{\circledR}\right)$. Pertinent literature was searched for, uncovered, and critically assessed to determine the comparative risk of arrhythmias or death between citalopram and escitalopram. No data were found directly addressing our clinical question by evaluating the two antidepressants for their comparative risk. However, several randomized and crossover trials, as well as a cohort study, were found to address questions regarding the cardiovascular risk of each agent. Available studies indicate a dose-related lengthening of the QT interval with both antidepressants, suggesting that these agents should be used at lower doses in patients with risk factors for arrhythmias. Overall, the evidence suggests that there is no clear advantage to using escitalopram in place of citalopram to minimize the risk of QT prolongation resulting in fatal arrhythmias.
\end{abstract}

Citalopram and escitalopram have been the subject of several Health Canada and U.S. Food and Drug Administration (FDA) advisories issued since August $2011 .^{1-5}$ They raise concern over the potential for these agents to cause QT prolongation. Excessive lengthening of the heart rate-corrected QT (QTc) interval may in rare cases induce Torsade de Pointes (TdP), a tachyarrhythmia associated with ventricular fibrillation, cardiac arrest, and in about 10 to $17 \%$ of cases, death. ${ }^{6,7}$ As patient heart rhythm is not continuously monitored and death occurs rapidly, measuring the rate of $\mathrm{TdP}$ and the resulting mortality is exceptionally difficult, especially in the outpatient setting. ${ }^{8}$

In adults, the normal QTc interval is $<430 \mathrm{msec}$ in males and $<450 \mathrm{msec}$ in females. ${ }^{9}$ Prolongation above 450 msec and $470 \mathrm{msec}$ in males and females, respectively, has been estimated to be associated with a two- to threefold increased risk of sudden cardiac death. ${ }^{8-10}$ Risk factors for QTc prolongation or arrhythmias include taking multiple QTc prolonging medications, age greater than 65 years, heart disease, electrolyte imbalances, and decreased systemic clearance of QTc-prolonging drugs. ${ }^{11-15}$

Case reports and cross sectional studies suggest that QTc prolongation with citalopram and escitalopram is dose-related. ${ }^{16,17}$ To address this concern, federal bulletins have recommend that daily doses of citalopram and escitalopram should not exceed $40 \mathrm{mg}$ and $20 \mathrm{mg}$, respectively, and that both agents be used at the lowest effective dose or avoided entirely in patients with risk factors. ${ }^{1,5}$
Escitalopram, the (S)-enantiomer of citalopram, is given at a lower dose than the racemic mixture while maintaining efficacy in the treatment of unipolar depression. ${ }^{18-20}$ The question as to whether this translates into a decreased risk of QTc prolongation remains unanswered. This report endeavors to determine if escitalopram has evidence to suggest its use in place of citalopram to lower the risk of QTc prolongation-related morbidity and mortality in adult patients with cardiovascular risk factors.

\section{Clinical Question}

In a middle-aged patient with the QTc prolonging risk factor of heart disease and recurrent major depressive disorder previously responsive to citalopram, is escitalopram an effective and safe option compared to citalopram to induce remission of depression while minimizing the risk of a serious and potentially fatal arrhythmia?

\section{Search Strategy}

The Cochrane Library, PubMed, EMBASE, and ClinicalTrials.gov were searched for studies pertinent to the clinical question. The keywords "citalopram", "serotonin uptake inhibitor", "long QT syndrome", and "cardiac arrhythmia" were used as medical search headings $(\mathrm{MeSH})$. Searches of titles and abstracts were conducted using keywords and additionally the terms "escitalopram", "selective serotonin reuptake inhibitor", "QTc", and "QT prolongation". Three assessors independently analyzed titles and abstracts of retrieved articles, and deemed 43 articles from EMBASE and three articles from PubMed, as potentially contributory to the question. These studies discussed 
QTc prolongation or arrhythmias in the context of citalopram or escitalopram.

We excluded non-English articles, trials reporting non-quantitative or unusable data ${ }^{21}$, and studies examining only acute overdoses (Figure 1 ). ${ }^{22}$ Searching references of the included articles yielded two additional studies that were relevant to the case ${ }^{23,24}$ Using Web of Science, we searched for relevant reviews and articles citing the selected studies. ${ }^{16,25,26}$

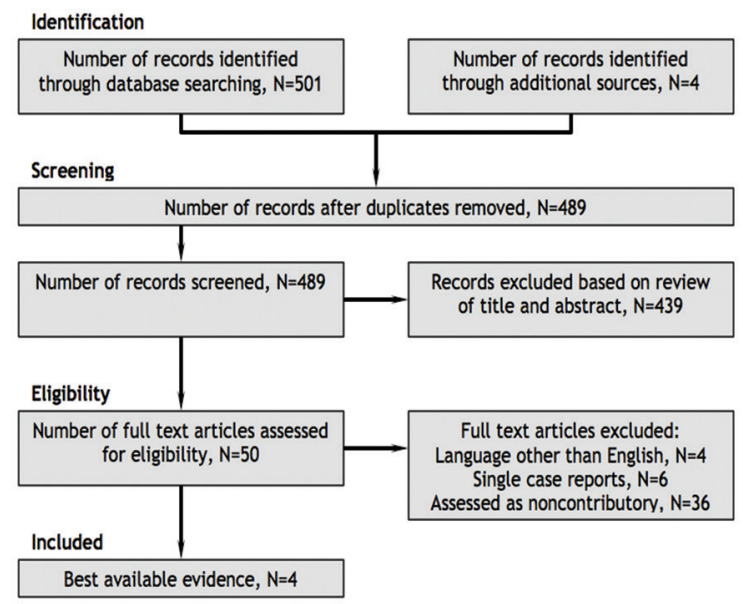

Figure 1. Search strategy and findings.

\section{Results}

Two randomized controlled trials (RCTs), two crossover studies, and one cohort study were considered as the best evidence addressing the clinical question (Table 1). The FDA summarized the results of two unpublished crossover studies assessing QTc prolongation with citalopram and escitalopram.5 RCTs by Hanash et al. ${ }^{27}$ and Lespérance et al. ${ }^{23}$ investigated escitalopram and citalopram therapy in patients with cardiovascular disease. The study by Leonard et al. assessed clinical outcomes of ventricular arrhythmia and sudden death (VA/SD) in a large cohort of antidepressant users. ${ }^{24}$

In each blinded crossover trial analyzed by the FDA, subjects received sequential therapy of increasing doses of citalopram or escitalopram, followed by moxifloxacin and placebo. ${ }^{5}$ Citalopram $20 \mathrm{mg} /$ day increased QTc duration by a mean of $8.5 \mathrm{msec}(90 \%$ CI, 6.2 to 10.8) from baseline. Escitalopram $10 \mathrm{mg} /$ day showed a mean change in QTc duration of 4.5 msec (90\% CI, 2.5 to 6.4). Based on the results, we calculated the standard deviation estimating the upper limit of change in QTc duration. While this may not accurately represent the risk in clinical practice, theoretically 1 of every 40 patients taking citalopram $20 \mathrm{mg} /$ day may have a QTc duration increase of 39.1 msec from baseline. Comparatively, 1 of 40 patients taking escitalopram $10 \mathrm{mg} /$ day may experience a QTc duration increase of $29.7 \mathrm{msec}$. Moxifloxacin $400 \mathrm{mg} /$ day showed a mean increase of $13.4 \mathrm{msec}$ ( $90 \% \mathrm{CI}, 10.9$ to 15.9$)$ in the citalopram study and $9.0 \mathrm{msec}(90 \% \mathrm{CI}$, 7.3 to 10.8 ) in the escitalopram study. The variability in moxifloxacin's effect indicates that the degree of QTc prolongation between citalopram and escitalopram may be more similar than suggested by these studies' results (Figure 2).

The FDA did not report cases of TdP or QTc changes with placebo. The inability to assess study details and patient characteristics, limits the inferences that can be drawn. The findings suggest a dose-dependent increase in QTc duration with both citalopram and escitalopram and neither agent appears safer at comparable doses. A $>30$ msec lengthening of the QTc interval or a duration $>500$ msec suggests an increased risk of arrhythmias including TdP. ${ }^{9}$ The subsequent risk of mortality in about 1 of 40 patients taking citalopram or escitalopram may be clinically significant for those with QTc prolonging risk factors.

In a 12-month RCT by Hanash et al., escitalopram 10 $\mathrm{mg} /$ day was compared to placebo in 240 adults with acute coronary syndromes (ACS) for prophylaxis of depression. ${ }^{27}$ ECG measurements showed no difference in QTc duration between the groups at six months and 12 months $(P>0.10)$. The overall incidence of major cardiac events was not significantly different between escitalopram and placebo ( $13.3 \%$ vs. $10.9 \% ; \mathrm{P}=0.59)$. Major cardiac events included recurrent acute coronary syndrome $(7.5 \%$ in the escitalopram group vs. $4.2 \%$ in placebo group; $\mathrm{P}=\mathrm{NS})$, need for acute revascularization (5.0\% vs. $2.5 \%$; $\mathrm{P}=\mathrm{NS})$, and death (5.0\% vs. $3.3 \%$; $\mathrm{P}=\mathrm{NS}$ ).

Lespérance et al. conducted a parallel group RCT comparing citalopram 20 to $40 \mathrm{mg}$ with matching placebo in 284 adults diagnosed with coronary artery disease (CAD) and depression. ${ }^{23}$ No statistically significant difference was found in mean QTc duration between citalopram and placebo after 12 weeks $(\mathrm{P}=0.18)$ and no cases of serious QTc prolongation (>525 msec) were reported. This confirms that the 1 in 40 risk of potentially clinically significant QTc prolongation is likely exaggerated from the theoretical assumptions extrapolated from the FDA studies. 


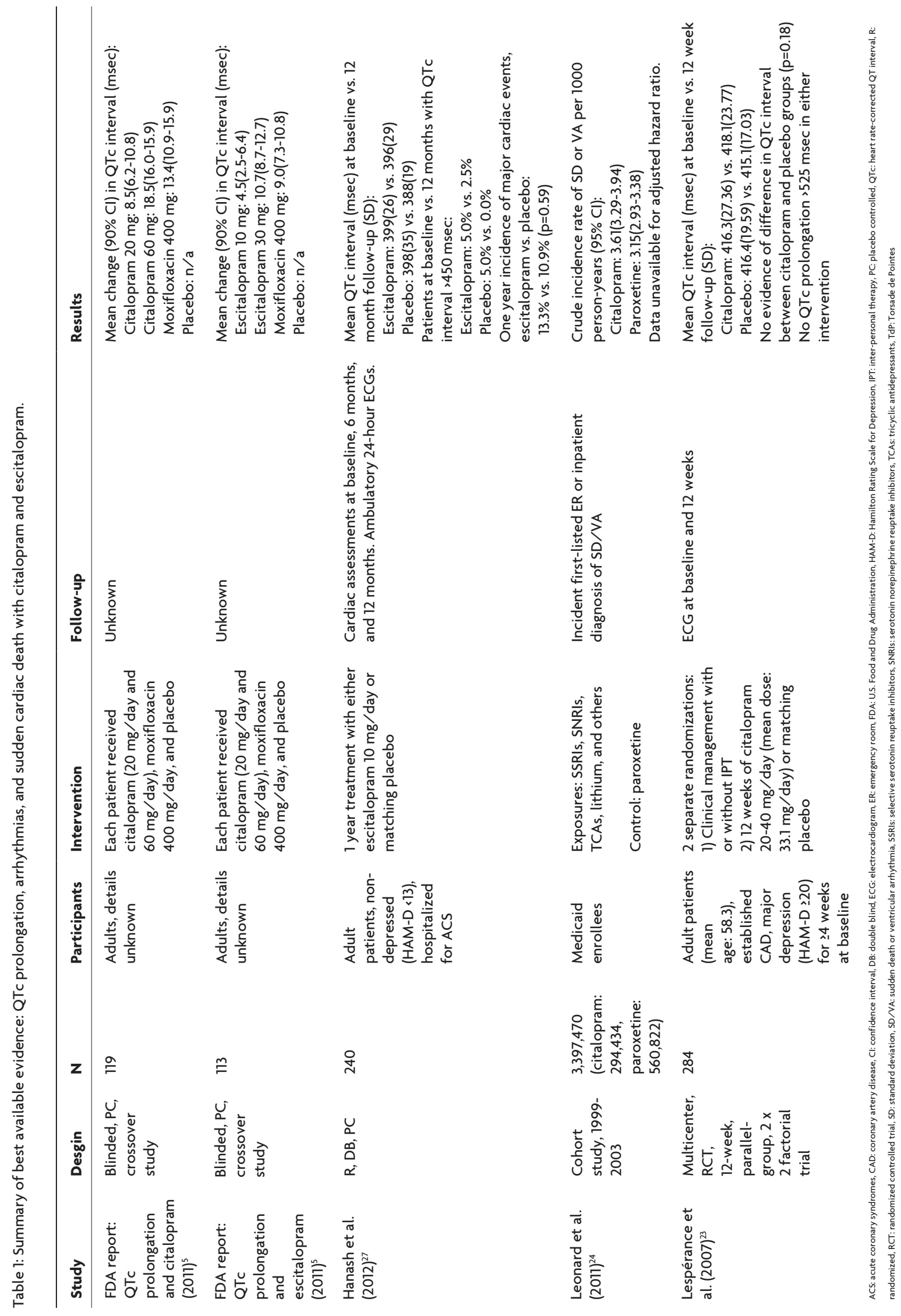




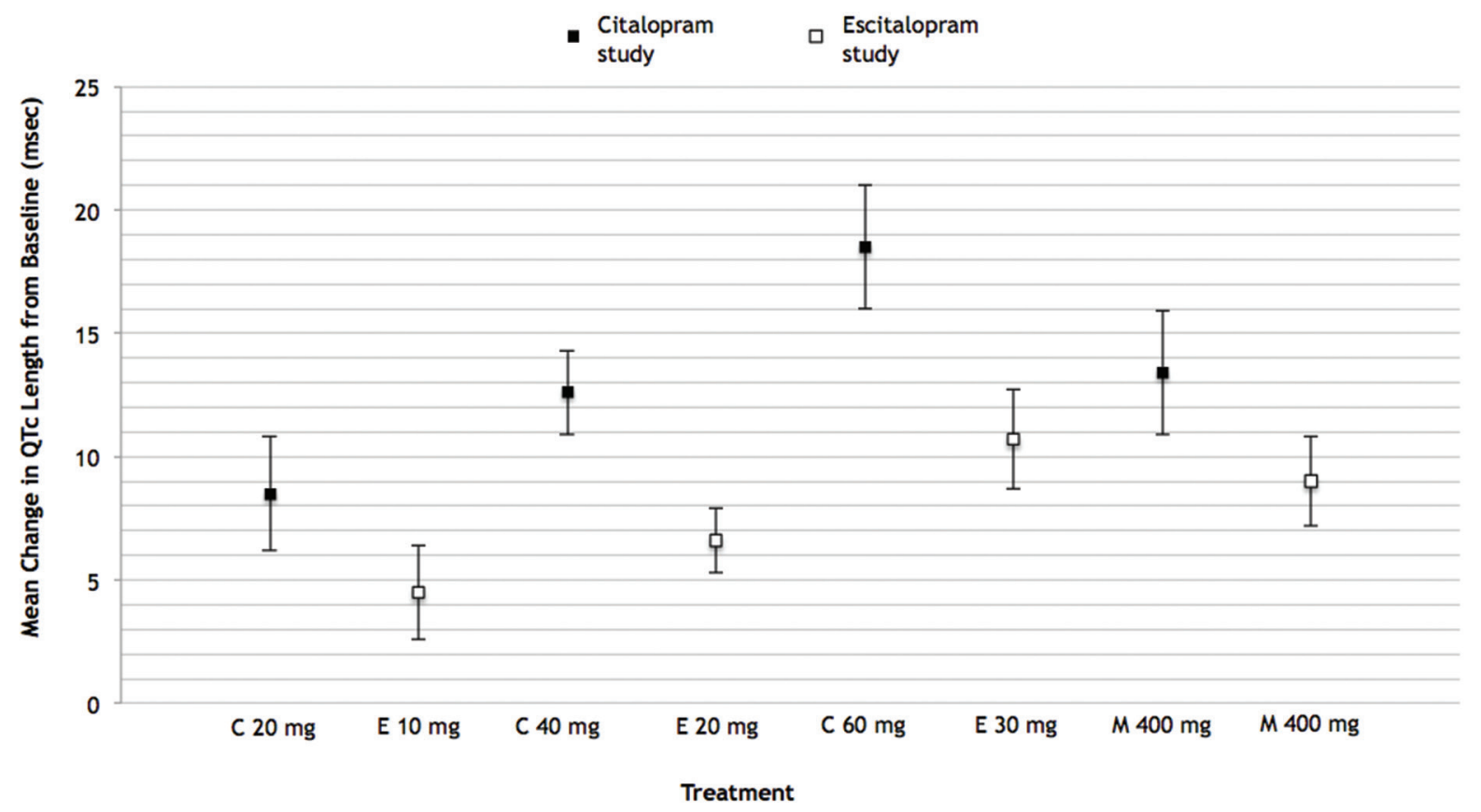

Figure 2. FDA study: $90 \%$ confidence intervals of mean QTc interval change with citalopram, escitalopram, and moxifloxacin. ${ }^{5}$

Hanash et al. and Lespérance et al. performed RCTs with adequate sample sizes to address efficacy of their intervention. ${ }^{23,28}$ However, when assessing the secondary outcome of clinical QTc prolongation both studies lacked adequate power, suggesting an overall inability to detect QTc changes at therapeutic doses. These studies contradict the FDA's findings that citalopram and escitalopram are associated with significant QTc changes. ${ }^{5}$

Leonard et al. completed a cohort study of over 3 million patients to examine the association between antidepressant exposure and hospital admission for VA/SD.24 Paroxetine was selected as the reference exposure to limit potential confounding by indication (depression) and due to its limited effect on the QTc interval. The unadjusted incidence rate ratio of 1.14 (95\% CI, 1.02 to 1.28 ) versus paroxetine suggested that exposure to citalopram may carry a slightly higher risk of VA/SD (Relative Risk=14\%). However, after adjusting for potential confounders, including age, sex, and nursing home residence, no difference in hazard was found (data unavailable). This analysis suggests that citalopram may not increase the risk of VA/SD compared to paroxetine's baseline incidence rate of 3.15 (95\% CI, 2.93 to 3.38) cases per 1000 patient years.

\section{Conclusion}

The available studies provide inconclusive data to suggest that either citalopram or escitalopram is more likely to cause fatal arrhythmias due to QTC prolongation. There is a lack of head-to-head trials comparing these agent's potential to cause QTc associated mortality at therapeutic doses.

The FDA's evidence favors low doses of both citalopram and escitalopram as both agents showed dose related QTc prolongation.5 Citalopram (20 mg/day) and escitalopram (10 mg/day) may theoretically increase the QTc interval by between 30 to $40 \mathrm{msec}$ in 1 of 40 patients, and therefore put those with underlying factors at risk of arrhythmias or sudden death.

Contrasting the FDA's findings, studies by Hanash et al.27 and Lesperance et al.23 found neither escitalopram $10 \mathrm{mg} /$ day nor citalopram 20-40 mg/day lengthened the QTc interval in patients at increased risk of arrhythmias due to heart disease. Similarly, Leonard et al.24 suggest that exposure to citalopram carries no greater risk of VA/SD compared to paroxetine, an antidepressant with a low propensity for QTc prolongation.

Considering the lack of evidence to suggest escitalopram has a safer cardiovascular profile than citalopram, a reasonable approach could be to consider citalopram 
$20 \mathrm{mg} /$ day as a safe and effective option. Based on the FDA's assessment, $20 \mathrm{mg} /$ day instead of $40 \mathrm{mg} /$ day is a prudent recommendation due to the dose related effect on the QTc interval. While not contraindicated, the tolerability and efficacy of escitalopram is unknown and the increased cost of therapy may compromise adherence and persistence with antidepressant therapy. Physicians should regularly screen for QTc prolonging risk factors and strongly consider an ECG at baseline and after six months of therapy. In addition to counselling and antidepressant therapy, patients should be advised to promptly report dizziness, palpitations, or syncope, as these symptoms may be indicative of cardiovascular conduction abnormalities. ${ }^{5}$

\section{Acknowledgements}

We would like to thank Dr. David Gardner for his thoughtful feedback on several drafts of this report.

\section{References}

1. "Antidepressant Cipralex (escitalopram): Updated information regarding dose-related heart risk." 7 May 2012. < http:// www.healthycanadians.gc.ca/recall-alert-rappel-avis/hcsc/2012/13674a-eng.php> (14 Nov 2012).

2. "Celexa (citalopram) - Association with Abnormal Heart Rhythms - For the Public." 30 Jan 2012. <http://www.healthycanadians. gc.ca/recall-alert-rappel-avis/hc-sc/2012/16887a-eng.php> (14 Nov 2012)

3. "Citalopram: Health Canada reviewing dose-related heart risk." 13 Oct 2011. <http://www.healthycanadians.gc.ca/recall-alertrappel-avis/hc-sc/2011/13549a-eng.php> (14 Nov 2012).

4. "FDA Drug Safety Communication: Abnormal heart rhythms associated with high doses of Celexa (citalopram hydrobromide)." 24 Aug 2011. <http://www.fda.gov/Drugs/DrugSafety/ ucm269086.htm> (14 Nov 2012).

5. "FDA Drug Safety Communication: Revised recommendations for Celexa (citalopram hydrobromide) related to a potential risk of abnormal heart rhythms with high doses." 28 Mar 2012. <http:// www.fda.gov/drugs/drugsafety/ucm297391.htm> (14 Nov 2012).

6. Shah RR. The significance of QT interval in drug development. $\mathrm{Br}$ J Clin Pharmacol 2002;54(2):188-202.

7. Salle P, Rey JL, Bernasconi P, Quiret JC, Lombaert M. Torsades de pointe. apropos of 60 cases. Ann Cardiol Angeiol 1985;34(6):381-

8. Drew BJ, Ackerman MJ, Funk M, Gibler WB, Kligfield P, Menon $\mathrm{V}$, et al. Prevention of torsade de pointes in hospital settings: a scientific statement from the american heart association and the american college of cardiology foundation. J Am Coll Cardiol 2010;55(9):934-47.

9. Committee for Proprietary Medicinal Products. Points to consider: the assessment of the potential for QT interval prolongation by non-cardiovascular medicinal products. London, UK: The European Agency for the Evaluation of Medicinal Products: Human Medicines Evaluation Unit; 1997.

10. Straus SM, Kors JA, De Bruin ML, van der Hooft CS, Hofman A, Heeringa J. Prolonged QTc interval and risk of sudden cardiac death in a population of older adults. J Am Coll Cardiol 2006;47(2):362-7.

11. Wenzel-Seifert K, Wittmann M, Haen E. QTc prolongation by psychotropic drugs and the risk of torsade de pointes. Dtsch Arztebl Int 2011;108(41):687-93.

12. Crouch MA, Limon L, Cassano AT. Clinical relevance and management of drug-related QT interval prolongation. Pharmacotherapy 2003;23(7):881-908.

13. Heist EK, Ruskin JN. Drug-induced arrhythmia. Circulation 2010;122(14):1426-35.

14. Malik M, Camm AJ. Evaluation of drug-induced QT interval prolongation: implications for drug approval and labelling. Drug Saf 2001;24(5):323-51.

15. Kanjanauthai S, Kanluen T, Chareonthaitawee P. Citalopram induced torsade de pointes, a rare life threatening side effect. Int J Cardiol 2008;131(1):e33-4.

16. Vieweg WV, Hasnain M, Howland RH, Hettema JM, Kogut C, Wood MA. Citalopram, QTc interval prolongation, and torsade de pointes. how should we apply the recent FDA ruling? Am J Med 2012;125(9):859-68.

17. Castro VM, Clements CC, Murphy SN, Gainer VS, Fava M, Weilburg JB. QT interval and antidepressant use: a cross sectional study of electronic health records. BMJ 2013;346:f288.

18. Ali MK, Lam RW. Comparative efficacy of escitalopram in the treatment of major depressive disorder. Neuropsychiatr Dis Treat 2011;7:39-4.

19. Cipriani A, Santilli C, Furukawa TA, Signoretti A, Nakagawa A, McGuire $\mathrm{H}$. Escitalopram versus other antidepressive agents for depression. Cochrane Database Syst Rev 2009;2:CD006532.

20. Culpepper L. Escitalopram: A new SSRI for the treatment of depression in primary care. Prim Care Companion J Clin Psychiatry 2002;4(6):209-14.

21. Rasmussen SL, Overo KF, Tanghoj P. Cardiac safety of citalopram: prospective trials and retrospective analyses. J Clin Psychopharmacol 1999;19(5):407-15.

22. Hayes BD, Klein-Schwartz W, Clark RF, Muller AA, Miloradovich JE. Comparison of toxicity of acute overdoses with citalopram and escitalopram. J Emerg Med 2010;39(1):44-8.

23. Lesperance F, Frasure-Smith N, Koszycki D, Laliberte MA, van Zyl LT, Baker B. Effects of citalopram and interpersonal psychotherapy on depression in patients with coronary artery disease: the canadian cardiac randomized evaluation of antidepressant and psychotherapy efficacy (CREATE) trial. JAMA 2007;297(4):36779.

24. Leonard CE, Bilker WB, Newcomb C, Kimmel SE, Hennessy S. Antidepressants and the risk of sudden cardiac death and ventricular arrhythmia. Pharmacoepidemiol Drug Saf 2011;20(9):903-1.

25. Howland RH. A critical evaluation of the cardiac toxicity of citalopram: part 1. J Psychosoc Nurs Ment Health Serv 2011;49(11):13-6.

26. Howland RH. A critical evaluation of the cardiac toxicity of citalopram: part 2. J Psychosoc Nurs Ment Health Serv 2011;49(12):13-6.

27. Hanash JA, Hansen BH, Hansen JF, Nielsen OW, Rasmussen A, Birket-Smith M. Cardiovascular safety of one-year escitalopram therapy in clinically nondepressed patients with acute coronary syndrome: results from the DEpression in patients with coronary ARtery disease (DECARD) trial. J Cardiovasc Pharmacol 2012;60(4):397-405.

28. Hansen BH, Hanash JA, Rasmussen A, Hansen JF, BirketSmith M. Rationale, design and methodology of a doubleblind, randomized, placebo-controlled study of escitalopram in prevention of depression in acute coronary syndrome (DECARD) Trials. 2009;10:20. 